\title{
A case of plurihormonal TSHoma presenting as meningitis
}

\section{Khan $\mathrm{S}^{1}$, Cudlip $\mathrm{S}^{2}$, Jafar-Mohammadi $\mathbf{B}^{1}$, Grossman $\mathrm{A}^{1}$, Ansorge $\mathbf{O}^{1}$, Pal $\mathrm{A}^{1}$}

1. Oxford Centre for Diabetes, Endocrinology and Metabolism, Churchill Hospital, Oxford University Hospitals NHS Foundation Trust

2. Department of Neurosurgery, John Radcliffe Hospital, Oxford University Hospitals NHS Foundation Trust

3. Department of Histopathology, John Radcliffe Hospital, Oxford University Hospitals NHS Foundation Trust

Introduction TSHomas are rare, accounting for $0.5-3 \%$ of pituitary tumours [1,2]. 70-80\% secrete only TSH, while $20-25 \%$

co-secrete predominantly prolactin and GH [1,3]. Almost all express somatostatin receptors, hence somatostatin analogues are routinely used as 2 nd line treatment after surgery [4]. Post operative cure is expected in more than half of macroadenomas \& almost all microadenomas [6]. We describe a recent interesting case of plurihormonal TSHoma from Oxford.

\section{Presentation}

A 22 year old lady with previous gestational diabetes presented with Haemophilus Influenza meningitis. MRI revealed an incidental pituitary macroadenoma extending into the right cavernous sinus and breaching the anteroinferior wall of the pituitary fossa with CSF leak. Clinically, she was mildly thyrotoxic but not obviously acromegalic or cushingoid.

Examination and visual fields were normal. Thyroid function showed raised T4 $[24.7 \mathrm{pmol} / \mathrm{L}$ (normal range 9-19 pmol/L)] and T3 [8.3 pmol/L (normal range 2.6-5.7 pmol/L)] with unsuppressed TSH [1.75 munit/L (normal range 0.3-4.2 munit/L)]. IGF-1 was also raised at $56.7 \mathrm{nmol} / \mathrm{L}$ (normal 12-50.1 nmol/L). OGTT (nadir GH $0.88 \mu \mathrm{g} / \mathrm{L}$ ) and GH day curve (mean GH $7.2 \mu \mathrm{g} / \mathrm{L}$ ) confirmed acromegaly along with possible TSH co-secretion.

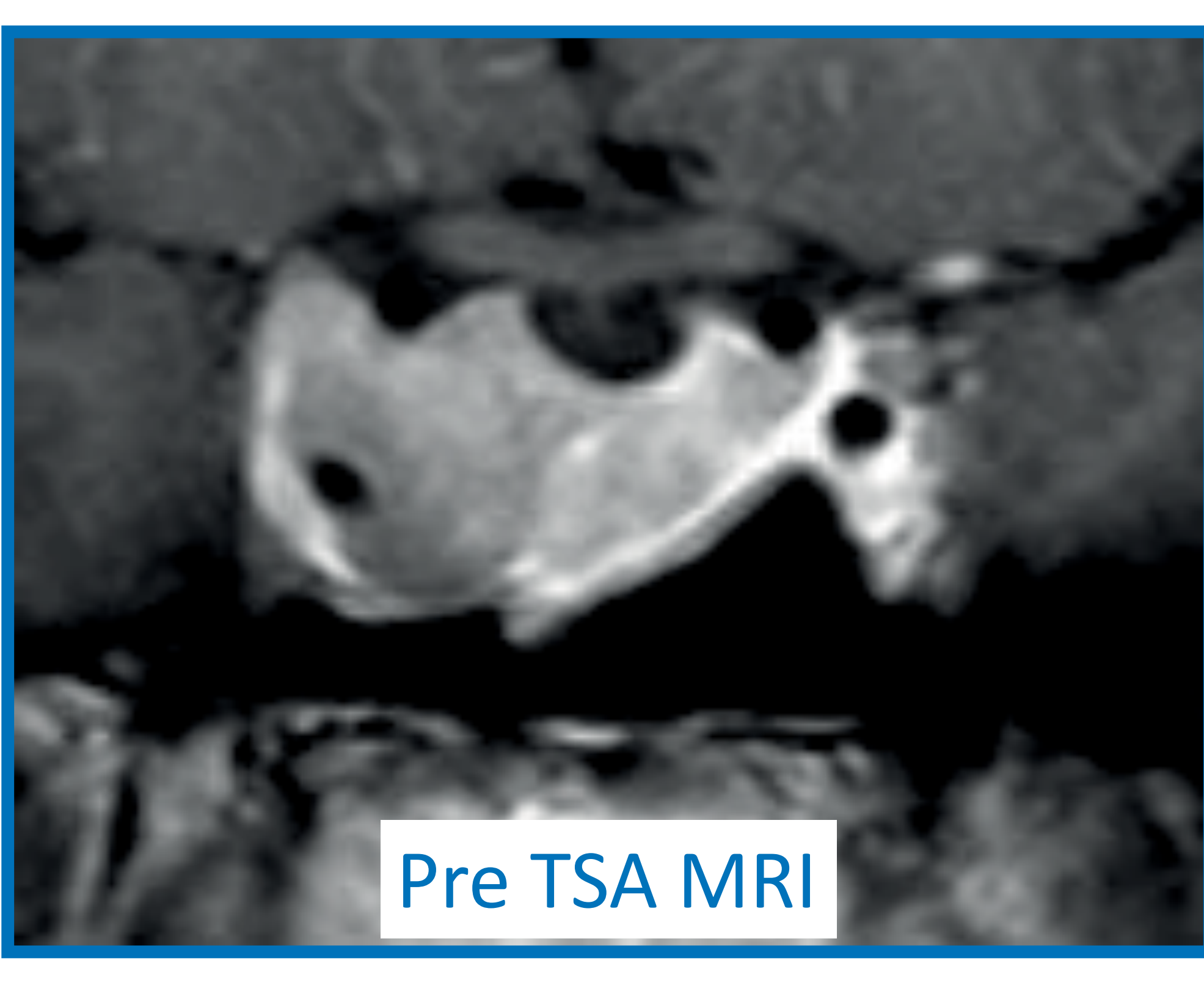

Pending trans sphenoidal surgery, Lanreotide was started which normalised thyroid Treatment function and IGF-1. However after 2 months treatment she developed a CSF leak and a second episode of meningitis.

After treatment and resolution of meningitis she had trans sphenoidal adenomectomy. A patch repair was fashioned to prevent further CSF leak on SSA treatment. Histology showed a plurihormonal atypical tumour with 30\% expression for GH, 1\% expression for TSH and Prolactin and MIB-1 index of 5-10\%. After surgery, T3, T4 and TSH remained in normal range at $5.1 \mathrm{pmol} / \mathrm{L}, 14.7 \mathrm{pmol} / \mathrm{L}$ and $1.68 \mathrm{munit} / \mathrm{L}$ respectively, however, IGF-1 level started to rise above the normal range at $56.4 \mathrm{nmol} / \mathrm{L}$. Hence she has been started on Lanreotide Autogel 120mg SC four weekly. This has lead to normalisation of IGF-1.

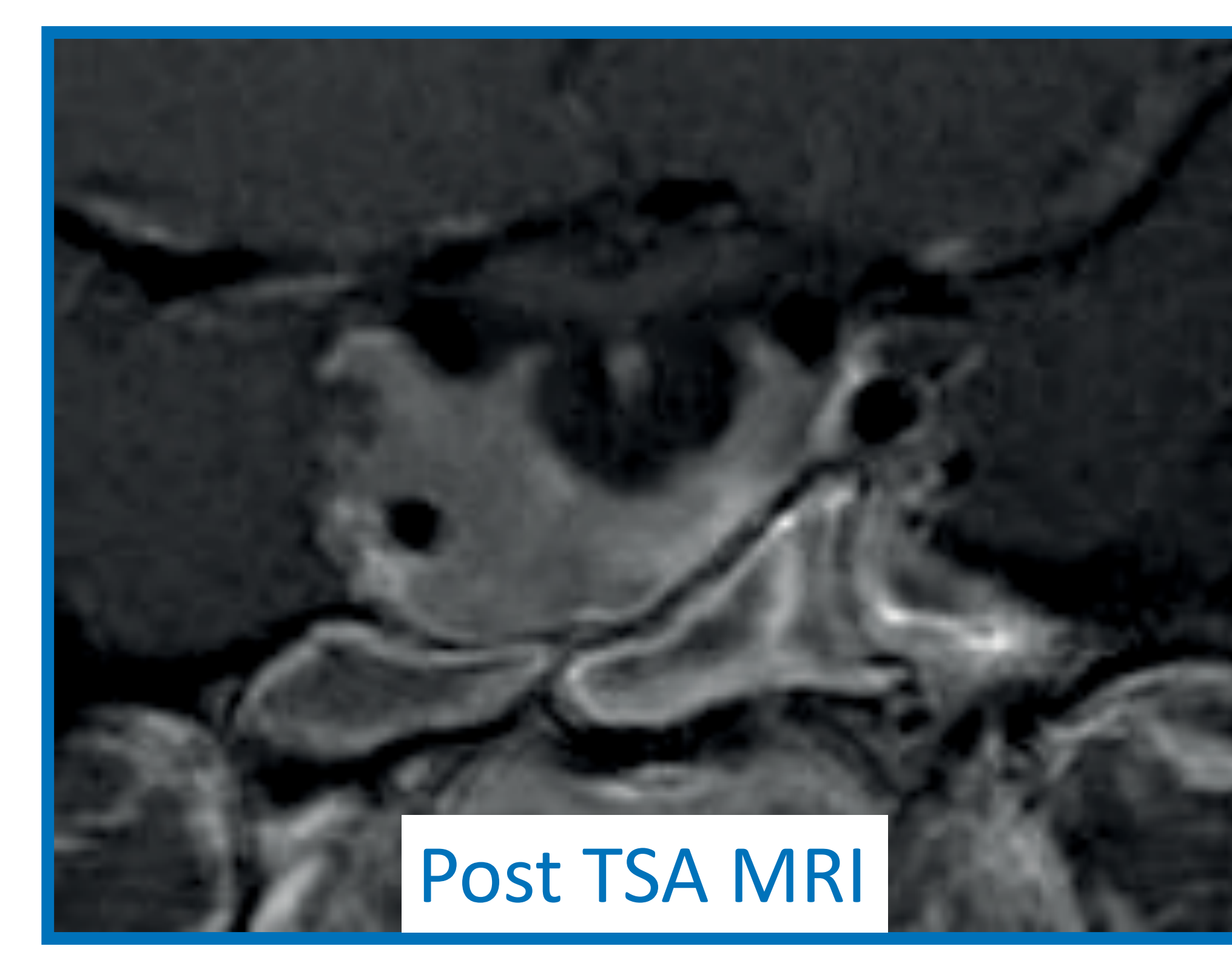

\section{Biochemistry}

\begin{tabular}{lllll} 
& Pre & 6 weeks & 12 weeks & On \\
& TSA & Post TSA & post TSA & SSA \\
\hline FT3 pmol/L & 8.3 & 5.1 & 5.3 & 1.9 \\
\hline FT4 pmol/L & 23.4 & 14.7 & 13.5 & 10.7 \\
TSH mU/L & 1.1 & 1.68 & 1.07 & 0.57 \\
IGF-1 nmol/L & 56.7 & 44.4 & 56.4 & 29.9 \\
$(12-50.1)$ & & & & \\
\hline
\end{tabular}
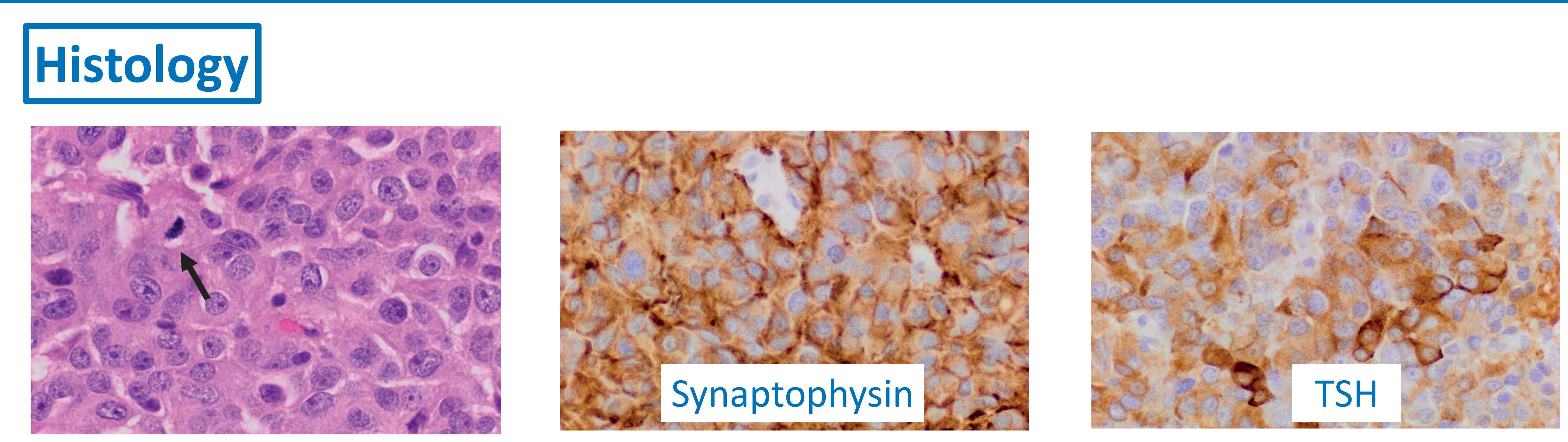

Histology demonstrated a neuroendocrine neoplasm with mitoses (arrow; usually absent in pituitary adenomas), diffuse synaptophysin expression (indicating presence of neurosecretory granules) and strong TSH expression.

\section{Conclusion \& clinical message}

This interesting case of TSHoma demonstrates an atypical presentation. It is noteworthy that this patient also developed CSF leak after pre-op somatostatin analogues which was probably due to tumour shrinkage. Histology confirmed the plurihormonal nature of the tumour as suspected from initial biochemistry. Clinically she was mildly thyrotoxic and did not have any acromegalic features, however histology showed $30 \% \mathrm{GH}$ expression compared to only $1 \%$ TSH. Due to cavernous sinus extension, cure was not achieved after surgery and second line treatment with somatostatin analogues is being used to maintain biochemical control and hopefully prevent further growth. Accurate diagnosis of TSHoma is important \& inappropriate treatments such as thyroid ablation \& surgery in cases of misdiagnosis are not uncommon $[5,6]$. Thyroid function tests should be analysed carefully and diagnosis should be questioned when response to treatment is not as predicted. A high index of suspicion should be maintained \& any instances of "inappropriate TSH" should be carefully tested further to rule out TSHomas [6,7].

References 1- Beck-Peccoz et al 2009. Endocrinol Metab; 23: 597-606. 2- Onnestam L, et al., 2013 JCEM; 98: 626-635. 3- Socin HV et al, 2003 Eur J Endocrinol ; 148:433-442. 4- Bertherat J et al 1992 JCEM 75: 540-546. 5- Losa $\mathrm{M}$, et al, 1996 JCEM; 81: 3086-3090. 6- Beck-Peccoz P et al., 2013 European Thyroid Journal. (2):76-82. doi:10.1159/000351007. 7- Koulouri O et al, Endocrinol Metab; 2013 Dec;27(6):745-62.

\section{The Oxford Centre} for Diabetes, Endocrinology and Metabolism 